\title{
Calibration of peripheral perception of shape with and without saccadic eye movements
}

\author{
Céline Paeye $^{1,2} \cdot$ Thérèse Collins $^{1} \cdot$ Patrick Cavanagh $^{1,2,3} \cdot$ Arvid Herwig $^{4,5}$
}

Published online: 11 January 2018

(C) The Psychonomic Society, Inc. 2018

\begin{abstract}
The cortical representations of a visual object differ radically across saccades. Several studies claim that the visual system adapts the peripheral percept to better match the subsequent foveal view. Recently, Herwig, Weiß, and Schneider (2015, Annals of the New York Academy of Sciences, 1339(1), 97-105) found that the perception of shape demonstrates a saccadedependent learning effect. Here, we ask whether this learning actually requires saccades. We replicated Herwig et al.'s (2015) study and introduced a fixation condition. In a learning phase, participants were exposed to objects whose shape systematically changed during a saccade, or during a displacement from peripheral to foveal vision (without a saccade). In a subsequent test, objects were perceived as less (more) curved if they previously changed from more circular (triangular) in the periphery to more triangular (circular) in the fovea. Importantly, this pattern was seen both with and without saccades. We then tested whether a variable delay between the presentations of the peripheral and foveal objects would affect their association - hypothetically weakening it at longer delays. Again, we found that shape judgments depended on the changes experienced during the learning phase and that they were similar in both the saccade and fixation conditions. Surprisingly, they were not affected by the delay between the peripheral and foveal presentations over the range we tested. These results suggest that a general associative process, independent of saccade execution, contributes to the perception of shape across viewpoints.
\end{abstract}

Keywords Visual perception $\cdot$ Perception and Action $\cdot$ Eye Movements

The retina is heterogenous: Visual acuity is high in foveal vision (which corresponds to two degrees of visual angle at

Electronic supplementary material The online version of this article (https://doi.org/10.3758/s13414-017-1478-3) contains supplementary material, which is available to authorized users.

Céline Paeye

celine.paeye@gmail.com

1 Laboratoire Psychologie de la Perception, UMR 8242, Paris Descartes University, Paris, France

2 Laboratoire Vision Action Cognition, EA 7326, Institut de Psychologie, Paris Descartes University, 92774 Boulogne-Billancourt Cedex, France

3 Department of Psychological and Brain Sciences, Dartmouth College, Hanover, NH, USA

4 Department of Psychology, Bielefeld University, 33501 Bielefeld, Germany

5 Cognitive Interaction Technology-Excellence Cluster, BielefeldUniversity, Bielefeld, Germany the center of gaze) and drops with eccentricity. As a consequence, an object will appear with a high definition in foveal vision but will be coarsely represented in the periphery. Therefore, there can be dramatic differences in the cortical representation of the same visual object, depending on gaze orientation. Nevertheless, most of the time, we are able to easily recognize an object in the periphery and perceive it as having the same shape as its foveal view.

This phenomenon of object constancy might be explained in terms of sensorimotor contingencies that the visual system would learn between eye movements and saccade-induced changes in the retinal inputs (Bompas \& O'Regan, 2006; O'Regan \& Noë, 2001). Past experience of associations between three components-(i) peripheral, coarse objects; (ii) saccade execution; and (iii) foveal, highly defined objects - would help the visual system to predict the appearance of an object after an eye movement (Herwig \& Schneider, 2014).

One way to test this hypothesis has been to manipulate the perceptual consequences of saccades in order to introduce a 
new sensorimotor contingency. In a typical experiment, a saccade target is altered during the eye movement. Due to the suppression of visual sensitivity during saccades (Ross, Morrone, Goldberg, \& Burr, 2001; Wurtz, 2008), this modification is mostly not noticed by the participants. The repeated presentations of such intrasaccadic changes led to object confusion across retinal positions (Cox, Meier, Oertelt, \& DiCarlo, 2005) or the recalibration of perceived target spatial frequency (Herwig \& Schneider, 2014) or size (Bosco, Lappe, \& Fattori, 2015; Valsecchi \& Gegenfurtner, 2016) during peripheral object recognition. Specifically, peripheral visual perception was biased toward the previously associated postsaccadic foveal input.

Recently, Herwig, Weiß, and Schneider (2015) found that peripheral perception of shape was biased by previously learned transsaccadic associations between peripheral and foveal information. More specifically, in a 30-min learning phase, participants were exposed to objects (e.g., green triangles, like in the example depicted in Fig. 1a), whose shape systematically changed during a saccade. At the same time,

C Learning - Fixation condition
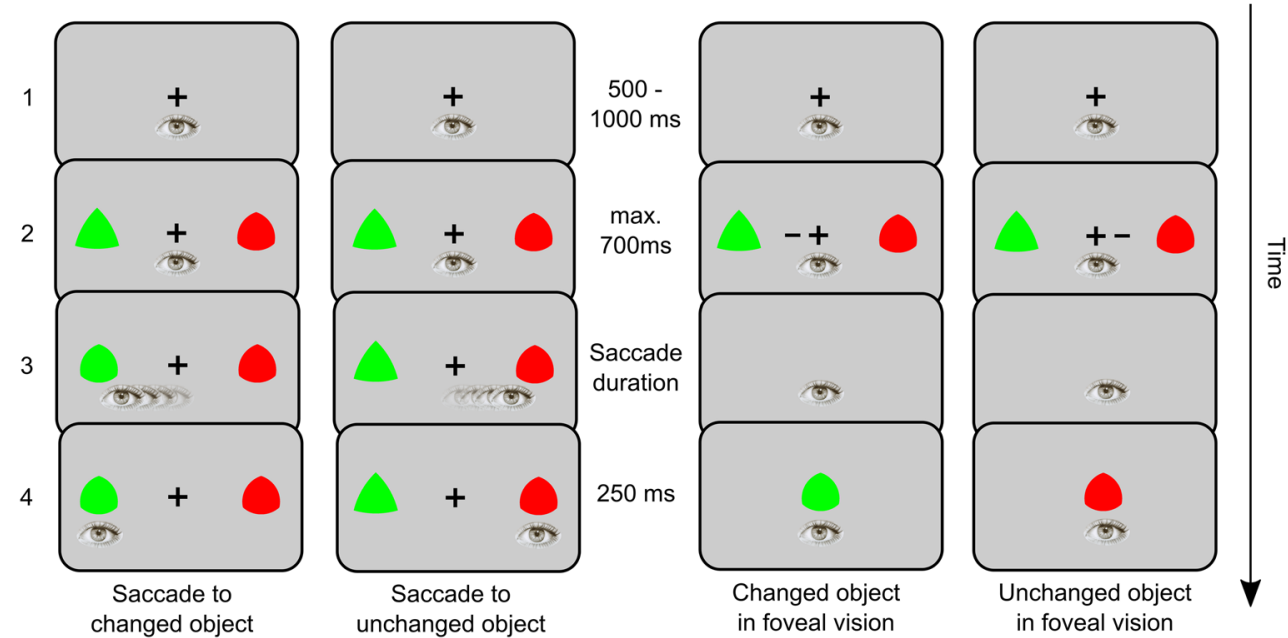

b Test Saccade condition

d Test Fixation condition

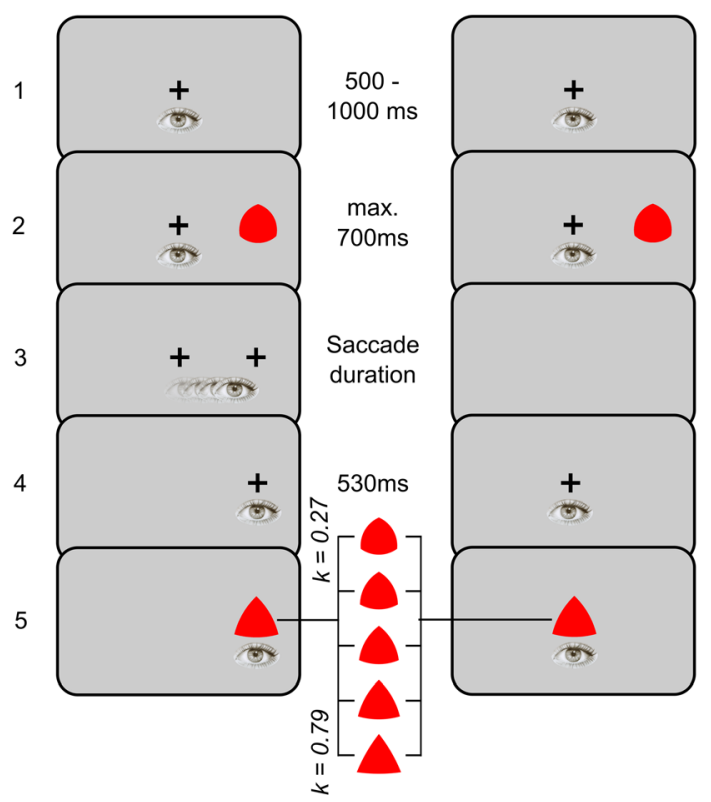

e Groups of participants

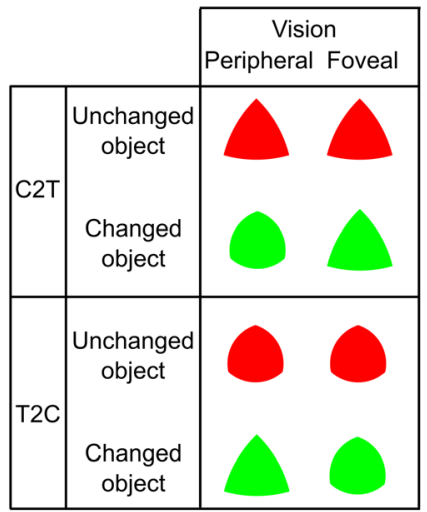

Fig. 1 Experimental protocol. a Structure of a learning trial in the saccade condition. Left column: saccade to the changed object; right column: saccade to the unchanged object. b Structure of a test trial in the saccade condition. c Structure of a learning trial in the fixation condition. Either the changed (left column) or unchanged (right column) object appears in foveal vision. $\mathbf{d}$ Structure of a test trial in the fixation condition. e Shape pairings used in each group of participants (in Experiment 1, the changed (unchanged) object was red (green) for half of the participants). (Color figure online) 
other objects of another color were not changed during the eye movement. In a subsequent test phase, objects were perceived as less (more) curved if they previously changed from more circular (triangular) in peripheral vision to more triangular (circular) in foveal vision. These results could be interpreted in terms of a learned contingency between the color of a given object seen before the saccade (in the periphery) and the perception of its shape after the eye movement (at the fovea). Such a contingency may result from an associative process that is specific to eye movements (i.e., that requires an intervening saccade to establish the correspondence between the two object views), or could result from a more general, movement-independent process.

In everyday life, the control of behavior by specific stimuli is an important aspect of how organisms adjust to their environment. This topic has been extensively studied by theoreticians of conditioning (e.g., Dinsmoor, 1995; Sidman, 2008). Not only humans but also animals (Guttman \& Kalish, 1956; Jarvik, 1956) and insects (Quinn, Harris, \& Benzer, 1974; Shafir, 1996) respond differentially in the presence of stimuli of discriminable colors. Several studies showed that visuomotor behavior can be influenced by learned contingencies involving colored stimuli. For instance, target selection in binocular rivalry (Marx \& Einhäuser, 2015) and attentional capture paradigms (Anderson, 2013; Awh, Belopolsky, \& Theeuwes, 2012; Hickey \& van Zoest, 2012) could be altered by learned contingencies in which reward was associated with stimuli of a given color.

We examined whether such a general associative process could also be involved in the transsaccadic learning reported by Herwig et al. (2015). To address this question, we first tested whether this learning specifically requires eye movements. We replicated Herwig et al.'s (2015) study and introduced a fixation condition in which participants experienced the same perceptual events as in the saccade condition but did not move their eyes. In this condition, stimuli were moved into different regions of their visual field to simulate the effects of the saccade that was not performed. We hypothesized that participants would associate a peripheral shape of a given color with a subsequent foveal shape in both fixation and saccade conditions.

Second, we manipulated the delay between the stimuli in the learning phase. Indeed, the temporal contiguity between two events is known to affect the ability to learn an association between them (Donahoe, Burgos, \& Palmer, 1993; Grice, 1948; Pavlov, 1927; Schultz, 2006). Therefore, if the hypothesis of an associative learning between presaccadic and postsaccadic information - or, more specifically, between peripheral and foveal stimuli-is true, differences between perceived shape of the changed and unchanged objects should be weakened at longer delays.

\section{Method}

\section{Stimuli}

Stimuli were generated using the Psychophysics Toolbox extensions for MATLAB (Brainard, 1997; Kleiner, Brainard, \& Pelli, 2007; Pelli, 1997). They were presented on a gray background with a mean luminance of $30 \mathrm{~cd} / \mathrm{m}^{2}$. The central fixation stimulus was a black cross $\left(0.3^{\circ} \times 0.3^{\circ}\right.$, line width 2 pixels). Peripheral and foveal objects were equiluminant (31 $\mathrm{cd} / \mathrm{m}^{2}$ ) red or green stimuli of different shapes.

The 2-D shapes were designed as follows. As in the study of Herwig et al. (2015), we used six intermediate shapes corresponding to the transformation of a circle (radius $=0.74^{\circ}$, curvature $k=$ 1) into an equilateral triangle (side length $=1.6^{\circ}$, curvature $k=0$ ). We used shapes with curvatures of $k=0.13,0.27,0.4,0.53,0.66$, 0.79 ( $k=0.74$ /radius). Therefore, the radii of the circles joining the vertices of the objects were respectively $5.29,2.74,1.85,1.40$, 1.12 , and $0.94^{\circ}$. We chose the stimuli that met the two following criteria. First, the distance between two vertices had to be smaller than $1.6^{\circ}$. Second, the differences between all the areas of the stimuli were minimized. Our six intermediate 2-D shapes had a mean area of $1.31 \mathrm{deg}^{2}(S D=0.01)$-while the areas of the circle $(k=1)$ and triangle $(k=0)$, as defined by Herwig et al. (2015), were respectively of 1.72 and $1.11 \mathrm{deg}^{2}$.

\section{Experiment 1}

\section{Procedure and design}

In this experiment, we tested whether the transsaccadic learning reported by Herwig et al. (2015) requires participants to execute eye movements to be able to associate a peripheral shape of a given color with a (possibly modified) foveal shape. Participants performed two experimental conditions: a saccade condition (an exact replication of Herwig et al., 2015) and a fixation condition, in which participants were required to look continuously at the center of the screen. In both conditions, a learning phase was followed by a test phase.

In the saccade condition, each trial of the learning phase started with the presentation of a central fixation cross (see Fig. 1a, Frame 1). After a random 500-1,000-ms fixation interval two objects (Frame 2), one green and one red, were presented $6^{\circ}$ to the right and left of fixation (the presentation side of each object was alternated pseudorandomly). Participants were free to choose which stimuli to saccade to and had up to $700 \mathrm{~ms}$ to execute the saccade before the trial was aborted with a speed up instruction presented. In addition, they were asked to look at each object about equally often. These stimuli differed in their shape, one being more triangu$\operatorname{lar}(k=0.27)$ than the other $(k=0.79)$. From the participants' point of view, the shape was task irrelevant. When the eye crossed a $1.5^{\circ}$ boundary around the fixation location, the 
saccade target object was either modified or remained unchanged (Frame 3). For the circular-to-triangular (C2T) group, the more circular object $(k=0.79$, hereafter referred to as the changed object) was systematically replaced with a more triangular one $(k=0.27)$ whereas the more triangular object $(k=$ 0.27 , hereafter the unchanged object) remained unchanged (see Fig. 1e). Conversely, for the triangular-to-circular (T2C) group, the more triangular object $(k=0.27)$ was replaced with a more circular one $(k=0.79)$, whereas the more circular object $(k=0.79)$ remained unchanged. That is, for both groups, the peripheral shapes always differed from each other, but the two objects had always the same shape when they were seen in foveal vision - since one of them was always changed during the saccade (see Fig. 1a, Frame 4). The associations of colors and object types (changed vs. unchanged) remained fixed for each participant but were counterbalanced across participants. Both peripheral objects remained on the screen for $250 \mathrm{~ms}$ and then disappeared. The next trial started $500 \mathrm{~ms}$ later with the presentation of the central fixation stimulus, and the fixation period was initialized as soon as the camera detected the eye within a $1.5^{\circ}$ circular zone around the fixation stimulus. This learning phase lasted half an hour and consisted of 240 trials. After each block of 48 trials, participants were presented with feedback indicating the number of times they looked at each stimulus, which enabled them to correct any too large discrepancy if needed.

In the test phase, after the initial 500-1,000-ms fixation interval (see Fig. 1b, Frame 1), only one object appeared at $6^{\circ}$, either to the right or to the left of fixation (Frame 2). The stimulus side and color (green or red) varied pseudorandomly. The curvature of this target object was 0.53 in two thirds of the trials. In the remaining third, we presented target objects with a curvature of $0.27,0.4,0.66$, or 0.79 . These catch trials were used to prevent participants from noticing the uniformity of the peripheral object's curvature. Participants had $350 \mathrm{~ms}$ to initiate a saccade to the target object (a latency $>350 \mathrm{~ms}$ aborted the current trial and triggered the instruction to speed up movement initiation). When the eye crossed a $1.5^{\circ}$ boundary around the fixation location (Frame 3), the target object was replaced with the fixation cross stimulus, that remained on for $530 \mathrm{~ms}$ (Frame 4). Then, a test object of same color as the target object appeared at the same (now foveated) location (Frame 5). Its shape was chosen randomly among the five curvatures of $k=0.27,0.4,0.53,0.66,0.79$. Participants were asked to adjust the shape of this test stimulus so that it matched the perceived shape of the previous stimulus presented in the periphery before the saccade. They did so by pressing the up or down arrow keys of a regular keyboard, which incrementally decreased or increased the curvature of the test objects by steps of $k=0.13$. Participants indicated their final choice by pressing the space bar. This 30-min test phase consisted of 192 trials counterbalancing saccade direction as well as object shape and color.
In the fixation condition, participants had to keep on looking at the central fixation location (if the eye crossed a $1.5^{\circ}$ boundary, the trial was aborted) in the learning phase (see Fig. 1c) as well as in the test phase (see Fig. 1d). To make the two experimental conditions comparable, the spatiotemporal parameters of the stimuli were the same as in the saccade condition, except the following changes. First, a cue (a black dash, $0.34^{\circ}$ length, line width 2 pixels) was presented simultaneously with the peripheral objects in the learning phase (see Fig. 1c, Frame 2). It was displayed $2^{\circ}$ either on the right or left of the fixation stimulus and signaled the color of the next stimulus (e.g., if it appeared on the same side as the green stimulus, a green object would appear at the fixation location). Second, the duration of the peripheral objects corresponded to the median saccadic latency (187-289 ms, median $238 \mathrm{~ms}$ ) measured in the saccade condition (see Fig. 1c-d, Frames 2). Third, in both phases, these stimuli were followed by a delay corresponding to the median saccadic duration $(36-46 \mathrm{~ms}$, median 38 ms; see Fig. 1c-d, Frames 3). Finally, the objects displayed subsequently were presented in foveal vision - that is, at the central fixation location (Frame 4). For changed objects, the foveal object was either more triangular (group $\mathrm{C} 2 \mathrm{~T}$ ) or more circular (group T2C) than the corresponding stimulus seen in the periphery. This aimed to reproduce the successive retinal stimulations experienced in the saccade condition. In both the learning and the test phases, there were as many trials as in the saccade condition.

Half of the participants performed the saccade condition first. In this case, the aforementioned time settings used in the fixation condition were retrieved from their own saccade data. The other participants began with the fixation condition. For these participants the time settings used in the fixation condition were retrieved from the saccade data of one of the participants who had started with the saccade condition.

\section{Participants}

Sixteen participants (13 females, three males; ages 20-29 years) performed the experiment. They were either students of the University of Paris Descartes or recruited via an advertisement through the French Relais d'Information sur les Sciences de la Cognition (RISC), which maintains a subject database. They had normal or corrected-to-normal vision. They received 20 euros for their participation. They came to the laboratory twice so that two experimental conditions were separated by at least 4 days. Experiments were in accordance with the principles of the Declaration of Helsinki and approved by the local ethics committee. Participants gave informed written consent prior to the experiment.

All participants were naïve as to the purpose of the study. They were not told that one of the stimuli would be changed transsaccadically during the acquisition phase. At the end of 
the experiment, they were asked whether they had noticed this change (see Supplemental Material).

\section{Apparatus}

Stimuli were displayed on a video monitor (Sony, $400 \times 300$ $\mathrm{mm}$, driven at $100 \mathrm{~Hz}$ ). Participants were seated in a dark room, and their head was stabilized by a chin and forehead rest at $63 \mathrm{~cm}$ from the screen. Eye movements were recorded with a video-based eye tracker (EyeLink 1000; SR Research, Ontario, Canada) and were sampled at $1000 \mathrm{~Hz}$. The eye tracker was calibrated via a 5-point procedure, which was repeated when necessary.

\section{Experiment 2}

We introduced a few changes in the procedure to test whether a delay between the peripheral and foveal stimuli during the learning phase would affect perceptual judgments made in a subsequent test phase. Only these modifications are listed below; otherwise, the procedure was identical to Experiment 1.

\section{Procedure and design}

Peripheral stimuli were presented at $9^{\circ}$, following Weiß and Herwig's (2015) experiment showing larger shape judgment differences at this eccentricity than at $6^{\circ}$. Since object color mapping had no influence in the previous experiment nor in the study of Herwig et al. (2015), the changed object in Experiment 2 was green for all the participants, whereas the red object never changed during an eye movement. The interstimulus delay introduced in the learning phase could be of 0 , 250,500 , or $1,000 \mathrm{~ms}$, depending on the participant. This delay remained fixed for each participant but varied between participants. The other time settings were the same as in the previous experiment.

In the fixation condition, for each participant, the time settings, the color and shape of stimuli, and their side of presentation were retrieved trial by trial from the saccade data of one of the participants who had already performed the saccade condition. Therefore, a participant who had completed the saccade condition first had to wait until her or his yoked participant also achieved the saccade condition in order to perform the fixation condition. In the learning phase of this condition, participants were asked to count the number of times they saw each stimulus at the central fixation location in each block of 48 trials. They had to report these numbers to the experimenter at the end of the block.

\section{Participants}

Thirty-two participants performed the experiment. Half of them (12 females, four males; ages $20-42$ years) were either students of the University of Paris Descartes or recruited in Paris via an advertisement. The other half (12 females, four males; ages 19-40 years) were recruited and performed the experiment in the Center of Excellence-Cognitive Interaction Technology of Bielefeld University. All participants had normal or corrected-to-normal vision. They received between 16 and 20 euros for their participation. The two experimental conditions were separated by at least 1 day. Experiments were in accordance with the principles of the Declaration of Helsinki and approved by the local ethics committees, and participants gave informed written consent prior to the experiment. All participants were naïve as to the purpose of the study.

\section{Apparatus}

In Paris, we used the same apparatus as in Experiment 1. In Bielefeld, stimuli were displayed on a video monitor (ViewSonic, Graphics Series G90fB, $360 \times 270 \mathrm{~mm}$, driven at $100 \mathrm{~Hz}$ ) situated at a distance of $71 \mathrm{~cm}$ from the participant. Eye movements were monitored with an EyeLink 1000 eye tracker (SR Research, Ontario, Canada) at $1000 \mathrm{~Hz}$ in both laboratories.

\section{Eye movement and response analyses}

For offline analyses of eye movements, ${ }^{1}$ we used the EyeLink parser to identify the onset and offset of saccades, using $30 \%$ velocity and $8,000 \% \mathrm{~s}^{2}$ acceleration thresholds. Saccades landing $3^{\circ}$ (in Experiment 1 ) or $5^{\circ}$ (in Experiment 2) further from the target or trials with blinks (overall, in $3.12 \%$ of trials, $S E M$ $=1.06$ in Experiment 1 and $4.50 \%$ of trials, $S E M=0.55$ in Experiment 2) were discarded from the analysis.

In the response analysis of the test phases, we first considered trials with a curvature of 0.53 . Student $t$ tests, 95\% confidence intervals, and analysis of variance techniques (ANOVA) were used to analyze the data. Where appropriate, Scheffe's post hoc method was used to further evaluate main effects and interactions. A Type I error rate of 0.05 was adopted for these analyses. In addition, we conducted Bayesian analyses using the open-source software JASP (JASP Team, 2017) to obtain 95\% credible intervals (95\% $\mathrm{CI}$ ) and to quantify evidence in favor of our hypotheses about shape judgments. In these analyses, the null model containing only the grand mean was compared to each of the models that could be created by including or not a main effect or interaction; results were expressed as Bayes factors of model comparisons $\left(\mathrm{BF}_{10}\right)$. Analyses of effects based on Bayesian model averaging also provided Bayes factor inclusion scores $\left(\mathrm{BF}_{\text {incl }}\right)$. The entire tables resulting from these Bayesian analyses are provided in the Supplemental Material (Tables S1a

\footnotetext{
${ }^{1}$ Raw data are available on Open Science Framework: https://osf.io/s39th
} 
and $\mathrm{S} 1 \mathrm{~b}$ for Experiment 1, and Tables $\mathrm{S} 2 \mathrm{a}$ to $\mathrm{S} 2 \mathrm{~b}$ for Experiment 2). The Cauchy prior width was set to the JASP default value of 0.707 .

In a second step, we analyzed shape judgments made in catch trials in which peripheral target objects had a curvature of $k=0.27,0.4,0.66$, or 0.79 (there were 16 trials per curvature for each participant). For each object type and each participant, a simple linear regression analysis was carried out using physical target object curvature (including 0.53 ) as the explanatory variable and mean judgment as the dependent variable. Individual slopes and coefficients of determination were then examined.

\section{Results}

\section{Experiment 1}

\section{Learning phase}

In the saccade condition, eye movements toward the changed object had a mean amplitude of $5.73^{\circ}(S E M=0.06)$ and those executed towards the unchanged object a mean amplitude of $5.7^{\circ}(S E M=0.07)$. These amplitudes did not differ from each other, $t(15)=1.07, p=.302$, nor did the corresponding median latencies of $223(S E M=6.65)$ and $222(S E M=6.51) \mathrm{ms}, t(15)$ $=0.65, p=.524$. The average saccade durations were also similar, both of $39 \mathrm{~ms}(S E M=0.77$ and 0.78$)$. Participants looked at the changed and unchanged stimuli in 50\% (SEM <
$0.01)$ of the trials. Objects' intrasaccadic change occurred on average $15 \mathrm{~ms}(S E M=0.75)$ before saccade offset (stimulus changes occurred after the saccade in $0.98 \%, S E M=0.32$, of trials). Eight participants reported noticing the changes. Their data were included in the analyses because our analyses did not show a difference between these participants that did notice and participants that did not notice the changes.

\section{Test phase}

In the saccade condition, eye movements had an average amplitude of $5.84^{\circ}(S E M=0.08)$, a median latency of $157 \mathrm{~ms}$ $(S E M=6.17)$ and a duration of $39 \mathrm{~ms}(S E M=0.89)$. Peripheral target stimuli disappeared on average $15 \mathrm{~ms}$ $(S E M=1.01)$ before saccade offset (and after it in $1.32 \%$, $S E M=0.57$, of trials, which were included in the analysis).

Figure 2a plots shape judgments of the changed objects against judgments of the unchanged objects for each participant and each condition. A first inspection of the judgments of the unchanged objects (compared to the actual curvature of 0.53 ) revealed a response bias in the T2C group (circles) only. Specifically, on average they judged the unchanged (circular) object as more triangular than it actually was, both in the saccade (mean $k=0.479), t(7)=44.603, p=.002,95 \% \mathrm{CI}$ $[0.454,0.505]$, and fixation $(k=0.503), t(7)=55.321, p=$ $0.022,95 \%$ CI $[0.482,0.525]$, conditions. In contrast, shape judgments for the unchanged objects were more accurate in the C2T group (triangles), in the saccade $(k=0.534), t(7)=$ 45.394, $p=.759,95 \%$ CI [0.506, 0.562], and fixation $(k=$
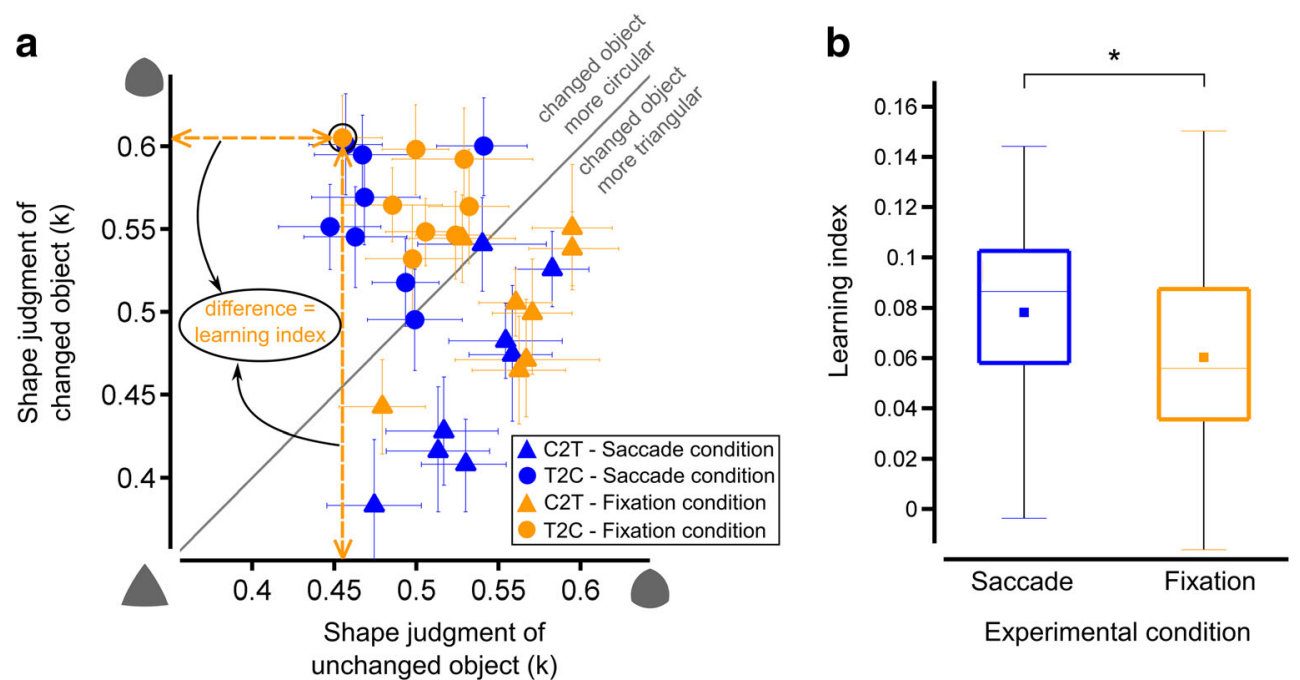

Fig. 2 Results of Experiment 1. a Shape judgment of changed object as a function of shape judgment of unchanged object for each participant and each experimental condition. The higher the curvature $(k)$, the more circular objects appeared to be. Example of learning index computation is given for one participant of the T2C group, in the fixation condition (black circle). Error bars: 95\% bootstrap confidence intervals. Each group can be clearly distinguished from the other: changed objects are judged as more triangular than unchanged objects in the $\mathrm{C} 2 \mathrm{~T}$ group whereas they are judged as more circular in the T2C group. b Boxplots of learning indices (i.e., judgment differences) in each experimental condition (this index was multiplied by -1 for the $\mathrm{T} 2 \mathrm{C}$ group). Squares: mean learning indices. $* p<.05$. Mean learning index was slightly but significantly higher in the saccade than in the fixation condition. Importantly, in each condition, this index was greater than zero, indicating that, overall, shape judgments of changed objects were biased toward foveal inputs associated in the preceding learning phase. (Color figure online) 
$0.557), t(7)=41.479, p=.082,95 \%$ CI $[0.525,0.589]$, conditions. However, this bias in the $\mathrm{T} 2 \mathrm{C}$ group is hard to interpret. One possibility is that it might be due to the curvature of the foveal object seen during the learning phase, leading to visual adaptation. But in this case, the asymmetry between groups is difficult to explain. A second possibility is that biases in the unchanged object might simply reflect the subjectivity of curvature judgments. Given that such a judgment bias might occur for various reasons not related to learning, we consider the comparison of the unchanged object, acting as a baseline measurement, with the changed object as a direct test of learning. We hypothesized that in each group the new association between peripheral and foveal objects would have an opposite effect on judgments of the changed objects.

Indeed, the data scatter into two groups, showing that the new association of foveal and peripheral inputs of the changed object had a specific, directional effect. On the one hand, data from the $\mathrm{C} 2 \mathrm{~T}$ participants are below the equality line (except one pair of blue and orange points whose $95 \%$ confidence intervals crossed this line), indicating that, overall, this group judged the changed object as more triangular than the unchanged object. On the other hand, data from the T2C group (circles) are above equality line (except two pairs of blue and orange points), indicating that overall these participants judged the changed object as more circular than the unchanged object.

To analyze changes in perceptual judgments, we computed a learning index for each participant and each condition. This index corresponded to the signed difference between the curvature judgments of the unchanged objects and those of the changed objects - these items differed only in their color. Figure 2a presents an example for one participant in the T2C group in the fixation condition (black circle). This participant perceived the changed object (which became more circular during the learning phase) as more circular than the unchanged object - specifically, her learning index was 0.48 $0.61=-0.13$.

Figure $2 \mathrm{~b}$ summarizes the results of this first experiment. The learning index of each participant of the T2C group was multiplied by -1 in order to be combined with that of the $\mathrm{C} 2 \mathrm{~T}$ group (therefore, the final learning index of the aforementioned participant was +0.13 ). Thus, in both groups, a positive value indicated that the judgment of the changed object was biased in the direction of previously associated foveal input, whereas a negative value indicated a judgment shift in the reverse direction. Overall, we found that the perceived shape of the changed object seen in the periphery was biased toward the foveal shape associated during the previous learning phase: the mean learning index was significantly greater than zero in both the saccade, $t(15)=7.33, p<.001, \mathrm{BF}_{10}=7717$ (where $\mathrm{BF}_{10}$ is the Bayes factor from the Bayesian one-sample test of comparison of shape judgments with zero), and fixation, $t(15)=6.20, p<.001, \mathrm{BF}_{10}=1371$, conditions. An analysis of distributions of the responses given to the $0.53 k$ target objects confirmed these conclusions (see Supplemental Material and Figure S1).

To compare the judgments made in the saccade and fixation conditions we ran a mixed analysis of variance on learning indices (i.e., shape judgment differences), with the withinsubjects factor condition (saccade vs. fixation) and the between-subjects factors change direction $(\mathrm{C} 2 \mathrm{~T}$ vs. T2C groups) and order (saccade performed first vs. fixation performed first). Because preliminary analysis revealed no effect of intrasaccadic change detection or object color mapping, we collapsed the data across these factors. We found no effect of change direction (i.e., group), $F(1,12)=0.117, p=.739, \mathrm{BF}_{\text {incl }}$ $=0.388$ (where $\mathrm{BF}_{\text {incl }}$ is the Bayes factor inclusion score from the analysis of effects), and removed this factor from our analysis. As illustrated in Fig. 2b, we observed higher learning indices in the saccade (mean $=0.078 k, S E M=0.10)$ than in the fixation condition (mean $=0.060 k, S E M=0.11), F(1,14)$ $=6.083, p=.027$, and this factor interacted with the order of the experimental conditions. $F(1,14)=4.789, p=.046, \mathrm{BF}_{10}$ $=1.978$ (where $\mathrm{BF}_{10}$ is the Bayes factor from the comparison between the model containing the two factors condition and order plus their interaction against the null model, see Fig. 3a and Supplemental Tables S1a and S1b).

A Scheffé post hoc analysis revealed that this interaction was driven by the high learning index observed in the participants who experienced the fixation condition first, when they performed the test in the saccade condition. For these participants, this test came second; the perceptual bias in their judgments of changed objects might have been established in the previous fixation condition and retained over the delay separating the two conditions. More importantly, there was no significant difference between the saccade and fixation conditions when they were performed first. That is, judgment differences in the fixation condition when participants began with this condition were not different from those observed in the saccade condition when participants began with this condition (both mean learning indices of 0.061, SEM $=0.15, p=$ .999 , in a Bayesian test of mean differences we obtained a Bayes factor of 2.338 in favor of the null hypothesis over the two-sided difference hypothesis). This clearly shows that the bias in peripheral perception of shape toward previously associated foveal inputs appeared even when participants did not execute a saccade to this peripheral object.

Finally, we analyzed the catch trials (namely, shape judgments of target objects with a curvature of $0.27,0.4,0.66$ or 0.79 ) to rule out decision biases and ensure that learned associations between peripheral and foveal objects really altered shape perception. If this was the case shape judgments should be scaled with the physical curvature of the peripheral objects. Figure 4 plots mean curvature judgments of each peripheral target object as a function of its status during learning (changed or unchanged object). In both groups and for each 


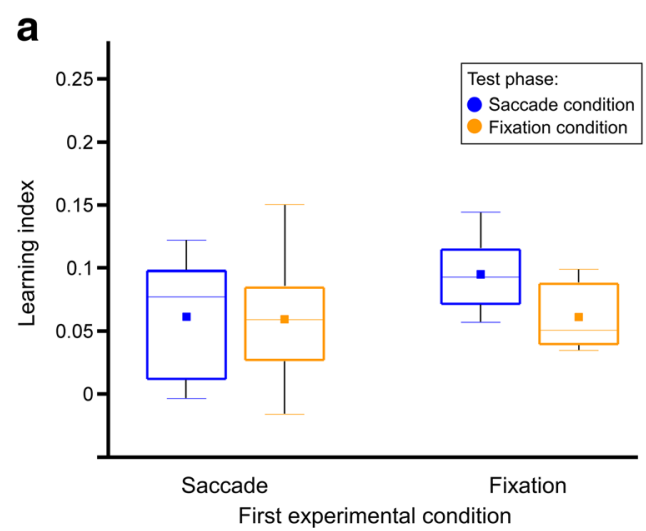

Fig. 3 Analysis of order effects (a) in Experiment 1 and (b) in Experiment 2. Box plots of learning index as a function of the first experimental condition that was performed. Squares: mean learning indices. There was no significant difference between the saccade condition when it was performed first (blue box plot on the left of the

participant, curvature judgments linearly increased with the physical curvature of target objects.
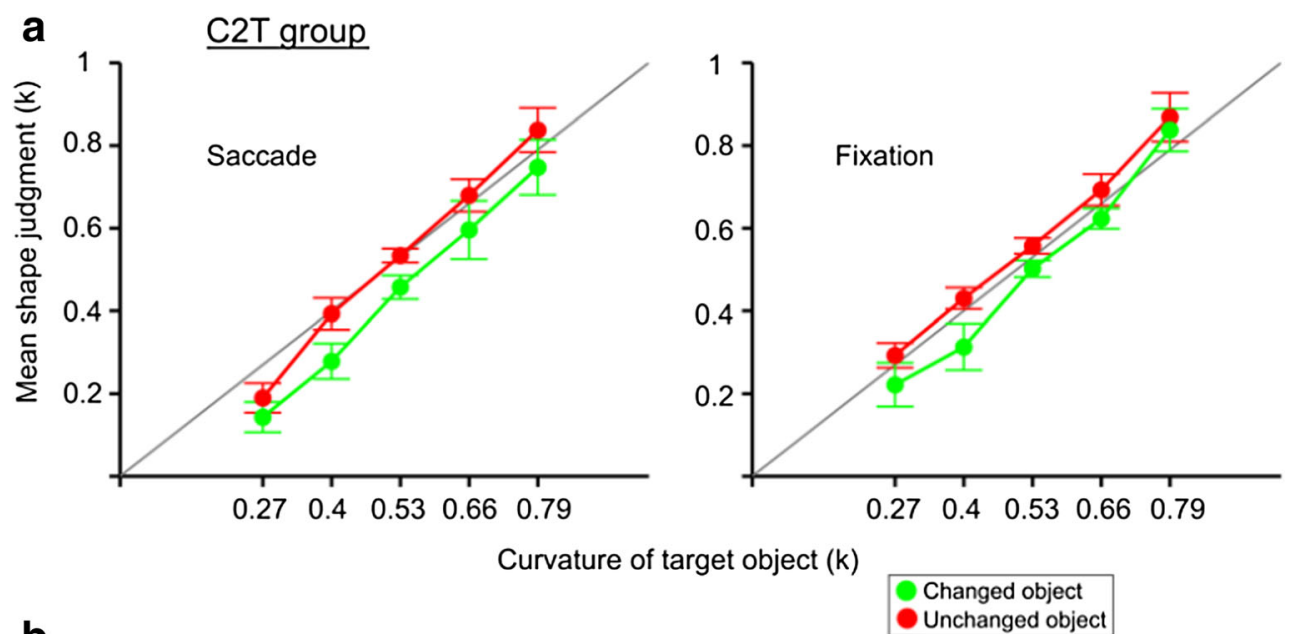

b
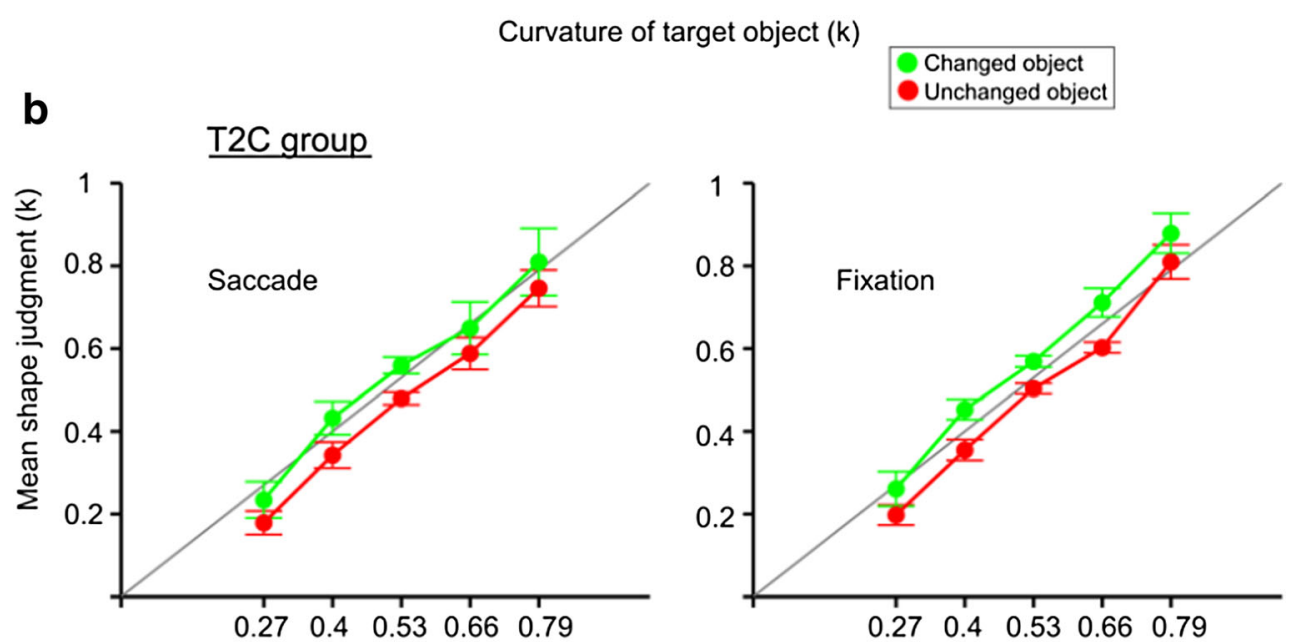

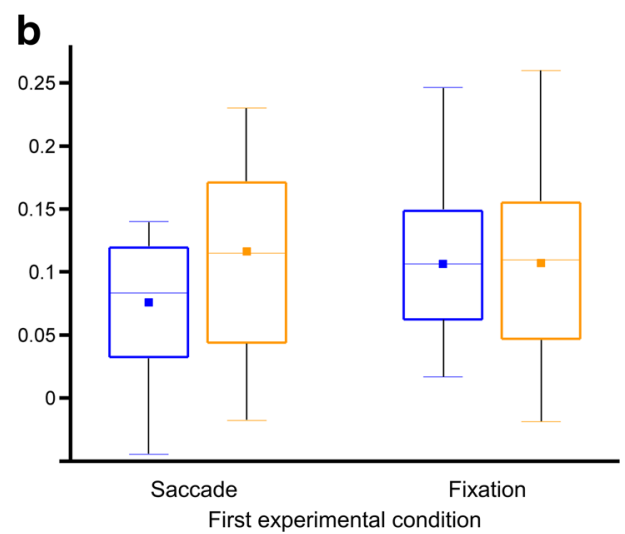

graphs) and the fixation condition when it was performed first (orange box plot on the right of the graphs), which indicates that shape judgments in both conditions were immediately equally affected by learned associations between foveal and peripheral objects. (Color figure online)

Table 1 summarizes the results of individual regression analyses for each group, each condition and each object type.

\section{Curvature of target object $(k)$}

Fig. 4 Mean shape judgments of peripheral target objects as a function of their physical curvature and their status during learning (i.e., changed or unchanged), in Experiment 1, for (a) the $\mathrm{C} 2 \mathrm{~T}$ and (b) the $\mathrm{T} 2 \mathrm{C}$ group, in

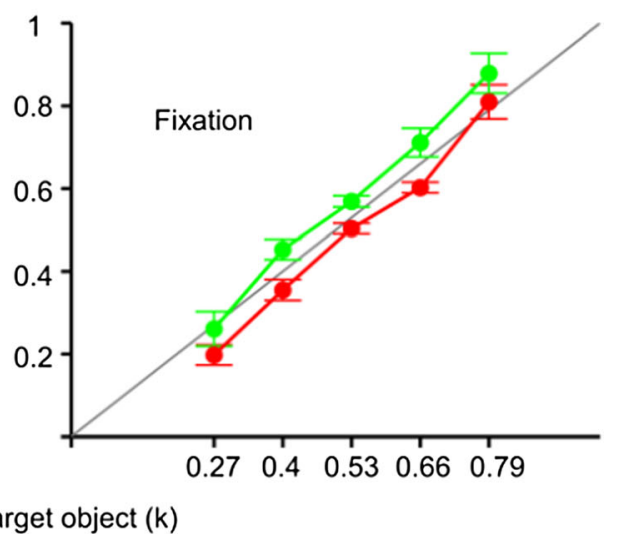

the saccade and fixation conditions. Error bars: SEM. Overall, shape judgments were highly correlated with the actual curvature of the target object. (Color figure online) 
Table 1 Mean slopes and $r^{2}$ (and $S E M$ ) of the individual linear regressions (predictor: physical curvature, dependent variable: shape judgments) averaged over participants of the same group (C2T or T2C), in each condition and for each object type

\begin{tabular}{|c|c|c|c|c|c|}
\hline & & \multicolumn{2}{|l|}{ Experiment 1} & \multicolumn{2}{|l|}{ Experiment 2} \\
\hline & & $\mathrm{C} 2 \mathrm{~T}$ group & $\mathrm{T} 2 \mathrm{C}$ group & C2T group & $\mathrm{T} 2 \mathrm{C}$ group \\
\hline \multirow[t]{4}{*}{ Saccade } & Changed object & $s=1.17(0.15)$ & $s=1.05(0.17)$ & $s=1.14(0.07)$ & $s=1.11(0.07)$ \\
\hline & & $r^{2}=.96(0.01)$ & $r^{2}=.89(0.06)$ & $r^{2}=.95(0.02)$ & $r^{2}=.95(0.01)$ \\
\hline & Unchanged object & $s=1.22(0.09)$ & $s=1.06(0.09)$ & $s=1.36(0.09)$ & $s=1.16(0.10)$ \\
\hline & & $r^{2}=.95(0.02)$ & $r^{2}=.95(0.02)$ & $r^{2}=.95(0.01)$ & $r^{2}=.97(0.01)$ \\
\hline \multirow[t]{4}{*}{ Fixation } & Changed object & $s=1.19(0.14)$ & $s=1.15(0.11)$ & $s=1.16(0.08)$ & $s=1.25(0.05)$ \\
\hline & & $r^{2}=.96(0.01)$ & $r^{2}=.96(0.02)$ & $r^{2}=.95(0.01)$ & $r^{2}=.97(0.01)$ \\
\hline & Unchanged object & $s=1.09(0.11)$ & $s=1.13(0.07)$ & $s=1.33(0.10)$ & $s=1.34(0.05)$ \\
\hline & & $r^{2}=.98(0.01)$ & $r^{2}=.97(0.01)$ & $r^{2}=.95(0.01)$ & $r^{2}=.95(0.01)$ \\
\hline
\end{tabular}

All individual slopes were significantly higher than zero (all $p s<.05$ ), and for each participant, each object and each condition, at least $92 \%$ of the variance was explained by the regression (except for one out of 64 cases, mean $=95.35 \%$ ). Therefore, our data do not seem to reflect a decisional bias to give a stereotyped response (e.g., the only object with a curvature of 0.53 seen in foveal vision) when one color is seen in the periphery. Rather they indicate that perception was truly affected by our learning phase.

In conclusion of this first experiment, we showed that perception of peripheral shapes was biased toward previously associated stimuli seen in foveal vision, and that this learning did not critically require eye movement. It is plausible that this learning results from a general associative process between stimuli. To further evaluate this possibility, we introduced a delay between the presentations of peripheral and foveal stimuli. With longer delays, associations between these shapes should be weaker than with shorter delays, and this might be reflected in smaller differences between judgments of unchanged and changed objects.

\section{Experiment 2}

\section{Learning phase}

We ran several analyses of variance to evaluate saccadic parameters as a function of object type (changed vs. unchanged) and delay of postsaccadic object reappearance. We found no significant effect of laboratory (Paris vs. Bielefeld), so we collapsed the data across this factor. Eye movements toward both the changed and unchanged objects had a mean amplitude of $8.4^{\circ}$ (both $\mathrm{S} E M=0.07$ ): Amplitude was affected neither by object type, $F(1,28)<0.001, p=.977$, nor by the delay between presaccadic and postsaccadic stimuli, $F(3,28)$ $=0.290, p=.835$. Similarly, saccadic duration was similar when participants made a saccade toward the unchanged and the changed objects (mean $=50 \mathrm{~ms}, S E M=0.71$ and 0.76 , respectively), $F(1,28)=3.673, p=.066$. Saccadic duration did not vary according to the interstimulus delay, $F(3,28)=$ $0.122, p=.946$. There was also no effect of object type, $F(1$, $28)=0.021, p=.885$, nor delay, $F(3,28)=1.323, p=.287$, on saccadic latencies, whose median was on average of $235 \mathrm{~ms}$ $(S E M=6.92)$.

Participants looked equally often at the unchanged and changed objects (respectively, in 50.12 and $49.88 \%$ of trials), and this was true for each interstimulus delay, $F(3,28)=$ $0.146, p=.931$. When no delay was programmed between the presaccadic and postsaccadic stimuli, their modification occurred on average $16 \mathrm{~ms}(S E M=1.55)$ before saccade offset (stimulus changes occurred after the saccade in $0.53 \%, S E M=$ 0.41 , of trials). Only one participant (in Paris) reported noticing that the green stimulus was changed (became more triangular) during the saccade.

\section{Test phase}

In the test phase of the saccade condition, saccadic latency, duration, and amplitude were normal for visually guided saccades - latency: $163 \mathrm{~ms}(S E M=4.42)$, duration: $50 \mathrm{~ms}$ (SEM $=0.56)$, and amplitude: $8.71^{\circ}(S E M=0.07)$. Peripheral target stimuli disappeared on average $17 \mathrm{~ms}(S E M=1.40)$ before saccade offset (and after it in $0.33 \%, S E M=0.26$, of trials, which were included in the analysis).

Like in Experiment 1, we found a response bias in curvature judgments of the unchanged objects (perceived as more triangular than it was) in the $\mathrm{T} 2 \mathrm{C}$ group, in the saccade (mean $k=0.443), t(15)=-4.210, p<.001,95 \%$ CI [0.399, 0.487], as well as in the fixation condition $(k=0.432), t(15)=-4.130, p$ $<.001,95 \%$ CI $[0.382,0.483]$. This bias was not observed in the $\mathrm{C} 2 \mathrm{~T}$ group, saccade condition: $k=0.538, t(15)=0.352, p$ $=.730,95 \%$ CI $[0.488,0.588]$; fixation condition: $k=0.545$, $t(15)=0.622, p=.544,95 \%$ CI $[0.498,0.596]$. Again, this bias observed in only one group is difficult to interpret, and we used the judgments of the unchanged objects as a baseline to 
compare judgments of the changed object by computing learning indices (i.e., shape judgment differences).

Overall, for each interstimulus delay, we found that judgments of the changed objects were biased toward the foveal shape associated during the learning phase: on the four plots of Fig. 5a, the data of the C2T group (triangles) can be clearly distinguished from that of the $\mathrm{T} 2 \mathrm{C}$ group (circles).

Mean learning indices were then analyzed as a function of the within-subjects factor condition (saccade vs. fixation) and the following between-subjects factors: change direction $(\mathrm{C} 2 \mathrm{~T}$ vs. T2C groups), order (saccade performed first vs. fixation performed first), and delay $(0,250,500$, or $1,000 \mathrm{~ms})$. Contrary to the first experiment, we found a main effect of change direction: mean judgment differences were larger in the $\mathrm{T} 2 \mathrm{C}$ group (mean $=0.126, S E M=0.012$ ) than in the $\mathrm{C} 2 \mathrm{~T}$ group (mean $=0.077, S E M=0.012), F(1,16)=7.802, p=$ $.013, \mathrm{BF}_{10}=3.390$. Given our difficulty to explain this effect of change direction, we performed two separate analyses, one for each group.

Learning indices, all greater than zero, were numerically higher in the fixation than in the saccade condition, but the
ANOVA showed no significant main effect of condition, group C2T: $F(1,8)=0.746, p=.413, \mathrm{BF}_{\text {incl }}=0.215$; group T2C: $F(1,8)=3.348, p=.105, \mathrm{BF}_{\text {incl }}=0.896\left(\right.$ where $\mathrm{BF}_{\text {incl }}$ are the Bayes factors inclusion scores from the analyses of effects). No other effect nor interaction was significant. In particular, there was no significant effect of delay on learning index, group C2T: $F(3,8)=0.929, p=.595, \mathrm{BF}_{\text {incl }}=0.261$; group T2C: $F(3,8)=0.764, p=0.545, \mathrm{BF}_{\text {incl }}=0.394$, as shown also by the overlapping $95 \%$ confidence intervals in Fig. 5b. The small decrease in learning index observed between an absence of delay between stimuli and a 250-ms interval did not reach significance. In addition, differences in shape judgments did not present any order effects, group C2T: $F(1,8)=0.307, p=.595, \mathrm{BF}_{\text {incl }}=0.301 ;$ group T2C: $F(1,8)=$ $0.081, p=0.783, \mathrm{BF}_{\text {incl }}=0.624$ (see Fig. 3b).

Finally, as in Experiment 1, we examined the distribution of responses to target objects with a curvature of $k=0.53$ (see Supplemental Material, Fig. S2) as well as catch trials. The latter were analyzed for each participant, in each condition, for each object type and change direction. We found that peripheral perception was still affected by the physical curvature of

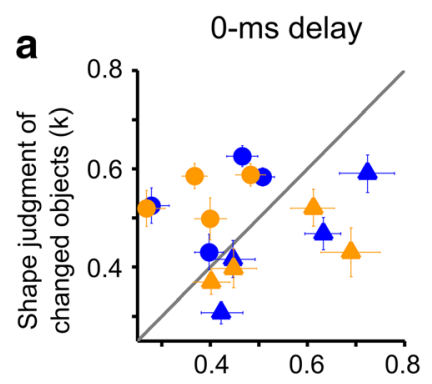

250-ms delay

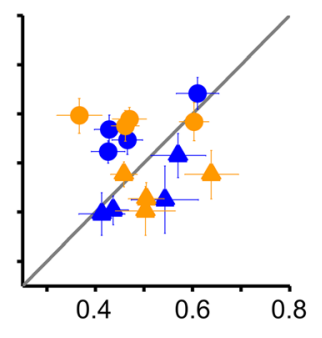

Shape judgment of unchanged objects $(k)$

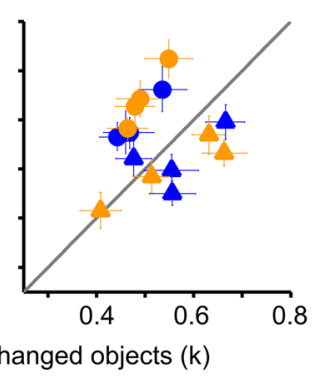

b

500-ms delay

b

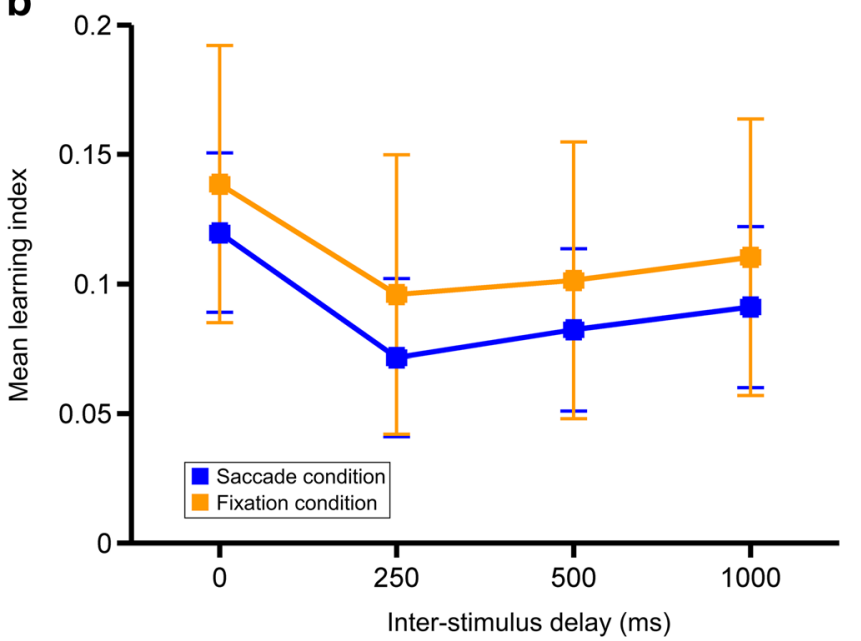

Fig. 5 Results of Experiment 2. a Individual shape judgment of the changed object as a function of the shape judgment of the unchanged object for each interstimulus delay $(0,250,500$, and 1,000 ms) and each experimental condition (saccade and fixation). The higher the curvature $(k)$, the more circular objects appeared to be. b Mean learning index (i.e., shape judgments differences) in each experimental condition (saccade and fixation) and for each delay between the presentations of the peripheral and foveal stimuli. Error bars: $95 \%$ confidence intervals. Differences between mean learning indices were not significant. (Color figure online) 
the peripheral target objects, as shown by the regression analyses presented in Fig. 6. All individual slopes (except one case out of 128) were significantly higher than zero (all $p \mathrm{~s}>.05$ ), and each regression explained an average of $95.61 \%$ of the variance (see Table 1).

\section{Discussion}

The two experiments reported here agree with previous studies on transsaccadic learning (Bosco et al., 2015; Herwig \& Schneider, 2014; Herwig et al., 2015; Valsecchi \& Gegenfurtner, 2016) in showing that perception of peripheral objects presented before an eye movement is biased by the peripheral-to-foveal changes experienced during a preceding training phase.

Our experiments extend these findings by showing that this learning is not eye-movement dependent. Indeed, similar perceptual effects were observed when objects were presented peripherally then foveally to participants maintaining their gaze at a central fixation. There has already been evidence suggesting that saccade execution might not be mandatory for such transsaccadic learning. First, although less pronounced, the descriptive pattern of results on the transsaccadic calibration of spatial frequency perception reported by Herwig and Schneider (2014, Experiment 1) resembled the effects observed in their Experiment 2 when no eye movement was required. Second, Valsecchi and Gegenfurtner (2016) reported reliable but less stable effects in peripheral size perception using object motion instead of eye movements to shift peripheral objects into foveal vision. Possibly in these studies the use of distinct groups of participants in the saccade versus fixation conditions could explain the different results.

The similarity of judgment differences observed with and without saccades suggests that bidirectional action-effect associations assumed by ideomotor theory (Elsner \& Hommel, 2001; Herwig, 2015a; Herwig, Prinz, \& Waszak, 2007; Hommel, 2009; Shin, Proctor, \& Capaldi, 2010) are not
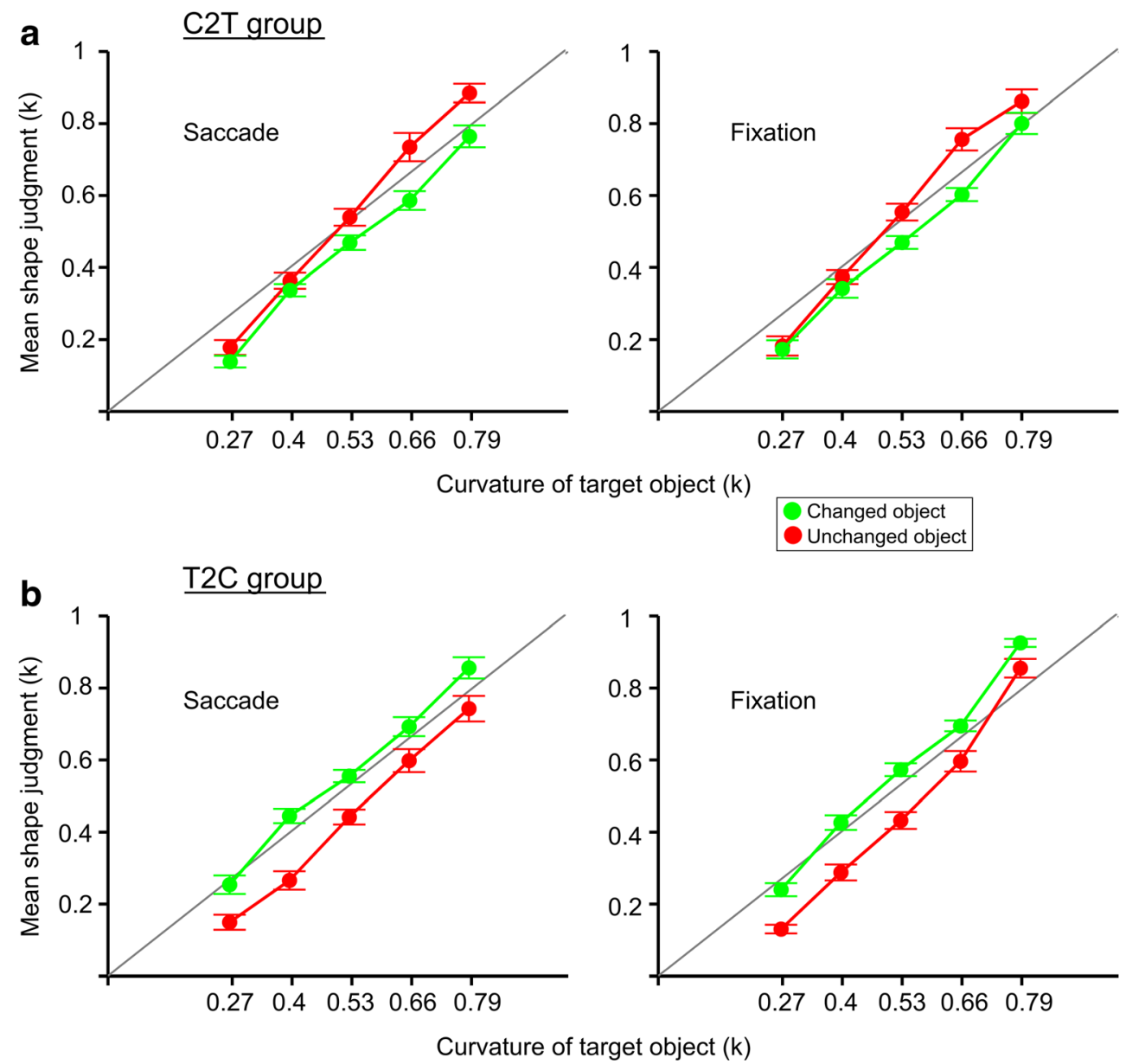

Fig. 6 Mean shape judgments of peripheral target objects (computed over all delays) as a function of object type (i.e., changed or unchanged), in Experiment 2 in the saccade and fixation conditions, for (a) the $\mathrm{C} 2 \mathrm{~T}$ and (b) the T2C group. Note the bias in shape judgments of the unchanged

objects in this group. Error bars: SEM. Overall, shape judgments were highly correlated with the actual curvature of the target object. (Color figure online) 
mandatory for the calibration of peripheral perception of shape. ${ }^{2}$ Instead, our results point to a more general associative learning process linking peripheral and foveal stimulus information that can be used to predict foveal appearance.

A candidate mechanism for associations between different retinal stimulations is a classical conditioning mechanism, a hypothesis that does not invoke highly specialized internal mechanisms. In a classical conditioning experiment, presentations of a conditioned stimulus (CS) are repeatedly followed by presentations of an unconditioned stimulus (US). As a result the CS comes to elicit a conditioned response that it did not elicit before (Pavlov, 1927; Rescorla \& Wagner, 1972). Presumably, in the present study the peripheral, colored - and coarse - stimulus (e.g., a green triangle) might function as a conditioned stimulus that signals the following, foveal shape of the same color (e.g., a green circle). The latter might act as an unconditioned stimulus eliciting the unconditioned response, namely, the perception of this object. Since it is seen in foveal vision, it is perceived in details and clearly identified. As a result of these CS-US pairings, a conditioned response would develop: the perception of peripheral shape toward the object seen in foveal vision. After our learning phase, when the changed object (green, in our example) is presented, this peripheral perception is biased (i.e., a peripheral triangle would appear more circular). This adaptive response of the visual system would be evoked by a stimulus of a given color in the periphery.

Several arguments support this hypothesis. First, a number of studies have shown that a visual sensation can develop as a conditioned response. This would be the case for color adaptation (Bompas \& O'Regan, 2006), perception in binocular rivalry (Marx \& Einhäuser, 2015), or the disambiguation of bistable stimuli (Haijiang, Saunders, Stone, \& Backus, 2006). Visual learning - that is, sensitivity changes for critical features of stimuli-may also arise through an automatic process similar to conditioning (Kim, Seitz, \& Watanabe, 2015; Seitz \& Watanabe, 2005). Interestingly, Seitz and colleagues showed that visual learning can also occur from unattended, task-irrelevant stimuli if these stimuli are temporally correlated with task-relevant ones (Seitz, Kim, \& Watanabe, 2009; Seitz \& Watanabe, 2003). Moreover, they found that such learning can lead to perceptual biases by which observers

\footnotetext{
${ }^{2}$ Current ideomotor theorizing assumes that the effective usage of bidirectional action-effect associations requires some form of contextualization (de Wit \& Dickinson, 2015; Hoffmann et al., 2007; Kiesel \& Hoffmann, 2004) because, usually, a given action-effect association works only under some but not under other contextual conditions. Thus, the required associative chain typically consists of three elements, namely, stimulus, response, and outcome ( $\mathrm{S}-\mathrm{R}-\mathrm{O})$ rendering the prediction of the outcome possible either via $\mathrm{R}-\mathrm{O}$ or via $\mathrm{S}-\mathrm{O}$ associations. This might also hold true for the current task, where a peripheral saccade target $(\mathrm{S})$ is sampled in foveal vision $(\mathrm{O})$ due to a saccade eye movement (R). Based on our data, the calibration of peripheral perception is mainly driven by $\mathrm{S}-\mathrm{O}$ associations, without any involvement of saccades (R).
}

misperceive their visual environment (Seitz, Nanez, Holloway, Koyama, \& Watanabe, 2005). Our findings suggest that this might be true for the establishment of transsaccadic correspondences: In our learning phase, a task-irrelevant shape was correlated with a task-relevant color (participants were asked to look equally often at the green and red objects), and this led to a bias in the conditioned perception of the peripheral shape. Second, the rewarding value of visual targets seen in foveal vision has been demonstrated in previous studies. The clear vision of targets can reinforce saccadic latencies, speed, and amplitudes in monkeys (Dorris, Pare, \& Munoz, 2000) and in humans (Collins, 2012; Madelain, Paeye, \& Wallman, 2011; Montagnini \& Chelazzi, 2005; Paeye \& Madelain, 2011; Schütz, Kerzel, \& Souto, 2014; Xu-Wilson, Zee, \& Shadmehr, 2009), as well as saccade sequences made during visual search (Paeye \& Madelain, 2014; Paeye, Schütz, \& Gegenfurtner, 2016).

A second candidate mechanism could also explain the differences between judgments of the changed and unchanged objects in both the saccade and fixation conditions. It is consistent with the object-based approach of transsaccadic correspondence (Deubel, Schneider, \& Bridgeman, 1996, 2002; Hollingworth, Richard, \& Luck, 2008; McConkie \& Currie, 1996) and requires specific attentional processes and visual short-term memory. More specifically, visual short-term memory would actively maintain spatial and feature information across the disruption caused by a saccade (Hollingworth et al., 2008; Schneider, 2013) or an occlusion (Hollingworth \& Franconeri, 2009)-like in our fixation condition. Based on spatiotemporal properties (Kahneman, Treisman, \& Gibbs, 1992) and/or surface feature information (Hollingworth \& Franconeri, 2009; Richard, Luck, \& Hollingworth, 2008), the visual system would decide whether to establish the correspondence between the stored and new information. If correspondence is established, predisruption and postdisruption information would be mapped to a single object representation (Moore, Mordkoff, \& Enns, 2007; Poth, Herwig, \& Schneider, 2015; Tas, Moore, \& Hollingworth, 2012) and even integrated in an optimal way (Ganmor, Landy, \& Simoncelli, 2015; Herwig, 2015b; Oostwoud, Marshall, \& Bays, 2015; Wolf \& Schütz, 2015).

The role of short-term memory in this updating of object representation across saccades has been highlighted, for instance, by Demeyer, De Graef, Wagemans, and Verfaillie (2010), who observed a shift of postsaccadic percept toward a presaccadic stimulus percept despite a 250 -ms blank between presaccadic and postsaccadic stimuli. This is consistent with the results of Noles, Scholl and Mitroff (2005), who showed that object representations can persist for at least 8 seconds. In addition, learning of associations between presaccadic and postsaccadic stimulations separated by $200 \mathrm{~ms}$ has been found to affect subsequent visual search by influencing feature prediction of upcoming foveal stimuli 
(Weiß, Schneider, \& Herwig, 2014). Our second experiment extends these results and shows that a shift of presaccadic percept toward a predicted postsaccadic view can be observed even when 1 second elapses between peripheral and foveal stimuli.

The absence of effect of long interstimulus delays is also compatible with an explanation in terms of classical conditioning. Indeed, it has been shown that CS-US associations can be established over a wide range of CS-US intervals largely exceeding 1 second (Delamater \& Holland, 2008; Kehoe, Cool, \& Gormezano, 1991; Marchand \& Kamper, 2000). Further research is needed to determine which learning mechanism can be responsible for the shift in the peripheral perception of changed objects' shape that we observed over delays as long as 1 second.

The appearance of a perceptual bias in the fixation condition suggests that attention might be another factor playing a key role in the calibration of peripheral perception. Unfortunately, our study does not allow us to describe more precisely the role of attention. First, our design did not guarantee that attentional prioritization of peripheral objects was the same in the two conditions. Indeed it is possible that in the fixation condition observers did not use the cue to prioritize one peripheral object over the other, whereas most of the attentional resources would have been shifted to the peripheral target in the saccade condition (Deubel \& Schneider, 1996; Henderson, Pollatsek, \& Rayner, 1989). Furthermore, we cannot be sure that the timing of attentional prioritization - if any - was comparable in both conditions. For instance, attention might have shifted from the periphery to the fovea about $50 \mathrm{~ms}$ before the saccade, provided predictive remapping processes took place (Duhamel, Colby, \& Goldberg, 1992; Rolfs, Jonikaitis, Deubel, \& Cavanagh, 2011). In contrast, in the fixation condition the shift of attention might have shifted from the periphery to the fovea at the onset of the foveal stimulus.

Nevertheless, in this later experimental condition we can be confident that the small number of colored items (two in the periphery and then one at the center) suddenly appearing on a uniform gray background - whatever their location - attract sufficient attention to enable participants to learn associations between peripheral and foveal items (Dinsmoor, 1995; Irwin \& Gordon, 1998; Schneider, 2013; Kruschke, 2003).

Following this reasoning, we can speculate that tests of the "reverse calibration" (Valsecchi \& Gegenfurtner, 2016) - that is, tests performed after a learning phase associating objects seen first in foveal and then in peripheral vision - would highlight biases in peripheral shape perception in the fixation condition. In contrast, and consistently with Valsecchi and Gegenfurtner's (2016) findings, we would expect to see no change in perceptual judgments about the shape of the presaccadic target in the saccade condition: Too few resources might have been allocated to the second (peripheral) stimulus after the saccade to induce this calibration (see also Experiment 3 of Ganmor, Landy, \& Simoncelli, 2015, for related findings on transsaccadic integration). Further experiments are needed to study more thoroughly the role of covert attention in the associative process taking place in the calibration of peripheral perception.

We acknowledge that the similarity of learning indices observed in the saccade and fixation conditions is not, alone, decisive evidence in support of a common learning process. Of course, in the saccade condition there is an additional mechanism which has to take into account the change in retinal coordinates induced by eye displacements, but this mechanism may be irrelevant to associative learning. In any case, our findings extend other studies reconsidering purely motor explanations of visual phenomena occurring around the time of a saccade. In this respect, our study joins others showing, for example, that saccadic suppression of displacement and perisaccadic compression of time and space can be observed in the absence of eye movements when visual input is masked (Zimmermann, Born, Fink, \& Cavanagh, 2014; Zimmermann, Fink, \& Cavanagh, 2013). Judgments about spatial relationships between objects (Deubel, Koch, \& Bridgeman, 2010; Hayhoe, Lachter, \& Feldman, 1991; Higgins \& Wang, 2010; Prime, Niemeier, \& Crawford, 2006), comparisons between stimuli (Irwin, 1991; Irwin, Zacks, \& Brown, 1990), and perceptual integration of presaccadic and postsaccadic stimuli (Paeye, Collins, \& Cavanagh, 2017; but see Fabius, Fracasso, \& Van der Stigchel, 2016; Ganmor et al., 2015; Wolfe \& Whitney, 2015) were comparable to those observed when they had to maintain steady fixation. Rather than being specific to the oculomotor system, these effects seem to constitute more general mechanisms that modulate perception across discontinuities in the visual scene. However, given their tremendous frequency of up to $4 \mathrm{~Hz}$, saccadic eye movements are arguably the most likely reason that peripheral stimuli end up in foveal vision. Thus, saccadic eye movements may be the prevalent condition in which these general learning mechanisms are harnessed in our everyday life.

Aknowledgements The research leading to these results received funding from the European Research Council under the European Union's Seventh Framework Program (FP7/2007-2013)/ERC Grant Agreement No. AG324070 to PC and by a grant of the German Research Council (Deutsche Forschungsgemeinschaft; DFG) Grant He6388/1-2 to A.H. The authors declare no competing financial interests.

\section{References}

Anderson, B. A. (2013). A value-driven mechanism of attentional selection. Journal of Vision, 13(3), 1-16.

Awh, E., Belopolsky, A. V., \& Theeuwes, J. (2012). Top-down versus bottom-up attentional control: A failed theoretical dichotomy. Trends in Cognitive Sciences, 16(8), 437-43. 
Bompas, A., \& O’Regan, J. K. (2006). More evidence for sensorimotor adaptation in color perception. Journal of Vision, 6(2), 5-5.

Bosco, A., Lappe, M., \& Fattori, P. (2015). Adaptation of saccades and perceived size after trans-saccadic changes of object size. The Journal of Neuroscience, 35(43), 14448-14456.

Brainard, D. (1997). The Psychophysics Toolbox. Spatial Vision, 10, 433-436.

Collins, T. (2012). Probability of seeing increases saccadic readiness. PLOS ONE, 7(11), e49454.

Cox, D. D., Meier, P., Oertelt, N., \& DiCarlo, J. J. (2005). « Breaking » position-invariant object recognition. Nature Neuroscience, 8(9), $1145-1147$

de Wit, S., \& Dickinson, A. (2015). Ideomotor mechanisms of goaldirected behavior. In T. S. Braver (Ed.), Motivation and cognitive control (Frontiers of cognitive psychology) (Chapter 7). New York: Routledge.

Delamater, A. R., \& Holland, P. C. (2008). The influence of CS-US interval on several different indices of learning in appetitive conditioning. Journal of Experimental Psychology: Animal Behavior Processes, 34(2), 202-222.

Demeyer, M., De Graef, P., Wagemans, J., \& Verfaillie, K. (2010). Parametric integration of visual form across saccades. Vision Research, 50(13), 1225-1234.

Deubel, H., Koch, C., \& Bridgeman, B. (2010). Landmarks facilitate visual space constancy across saccades and during fixation. Vision Research, 50(2), 249-259.

Deubel, H., \& Schneider, W. X. (1996). Saccade target selection and object recognition: Evidence for a common attentional mechanismVision Research, 36(12), 1827-1837.

Deubel, H., Schneider, W. X., \& Bridgeman, B. (1996). Postsaccadic target blanking prevents saccadic suppression of image displacement. Vision Research, 36(7), 985-996.

Deubel, H., Schneider, W. X., \& Bridgeman, B. (2002). Transsaccadic memory of position and form. Progress in Brain Research, 140, $165-180$.

Dinsmoor, J. A. (1995). Stimulus control: Part I. The Behavior Analyst, $18(1), 51-68$

Donahoe, J. W., Burgos, J. E., \& Palmer, D. C. (1993). A selectionist approach to reinforcement. Journal of the Experimental Analysis of Behavior, 60(1), 17-40.

Dorris, M. C., Pare, M., \& Munoz, D. P. (2000). Immediate neural plasticity shapes motor performance. Journal of Neuroscience, 20(1), 15 .

Duhamel, J. R., Colby, C. L., \& Goldberg, M. E. (1992). The updating of the representation of visual space in parietal cortex by intended eye movements. Science, 255(5040), 90-92.

Elsner, B., \& Hommel, B. (2001). Effect anticipation and action control. Journal of Experimental Psychology: Human Perception and Performance, 27(1), 229-240.

Fabius, J. H., Fracasso, A., \& Van der Stigchel, S. (2016). Spatiotopic updating facilitates perception immediately after saccades. Scientific Reports, 6, 1-11.

Ganmor, E., Landy, M., \& Simoncelli, E. (2015). Near-optimal integration of orientation information across saccadic eye movements. Journal of Vision, 15(12), 1306-1306.

Grice, G. R. (1948). The relation of secondary reinforcement to delayed reward in visual discrimination learning. Journal of Experimental Psychology, 38(1), 1-16.

Guttman, N., \& Kalish, H. I. (1956). Discriminability and stimulus generalization. Journal of Experimental Psychology, 51(1), 79-88.

Haijiang, Q., Saunders, J. A., Stone, R. W., \& Backus, B. T. (2006). Demonstration of cue recruitment: Change in visual appearance by means of Pavlovian conditioning. Proceedings of the National Academy of Sciences of the United States of America, 103(2), 483-488.
Hayhoe, M., Lachter, J., \& Feldman, J. (1991). Integration of form across saccadic eye movements. Perception, 20(3), 393-402.

Henderson, J. M., Pollatsek, A., \& Rayner, K. (1989). Covert visual attention and extrafoveal information use during object identification. Attention, Perception, \& Psychophysics, 45(3), 196-208.

Herwig, A. (2015a). Linking perception and action by structure or process? Toward an integrative perspective. Neuroscience \& Biobehavioral Reviews, 52, 105-116.

Herwig, A. (2015b). Transsaccadic integration and perceptual continuity. Journal of Vision, 15(16), 1-7.

Herwig, A., Prinz, W., \& Waszak, F. (2007). Two modes of sensorimotor integration in intention-based and stimulus-based actions. The Quarterly Journal of Experimental Psychology, 60(11), 1540-1554.

Herwig, A., \& Schneider, W. X. (2014). Predicting object features across saccades: Evidence from object recognition and visual search. Journal of Experimental Psychology: General, 143(5), 1903-1923.

Herwig, A., Weiß, K., \& Schneider, W. X. (2015). When circles become triangular: How transsaccadic predictions shape the perception of shape. Annals of the New York Academy of Sciences, 1339(1), 97105.

Hickey, C., \& van Zoest, W. (2012). Reward creates oculomotor salience. Current Biology, 22(7), 219-220.

Higgins, J. S., \& Wang, R. F. (2010). A landmark effect in the perceived displacement of objects. Vision Research, 50(2), 242-248.

Hoffmann, J., Berner, M., Butz, M. V., Herbort, O., Kiesel, A., Kunde, W. \& Lenhard, A. (2007). Explorations of anticipatory behavioral control $(\mathrm{ABC})$ : A report from the Cognitive Psychology Unit of the University of Würzburg. Cognitive Processing, 8, 133-142.

Hollingworth, A., \& Franconeri, S. L. (2009). Object correspondence across brief occlusion is established on the basis of both spatiotemporal and surface feature cues. Cognition, 113(2), 150-166.

Hollingworth, A., Richard, A. M., \& Luck, S. J. (2008). Understanding the function of visual short-term memory: Transsaccadic memory, object correspondence, and gaze correction. Journal of Experimental Psychology: General, 137(1), 163-181.

Hommel, B. (2009). Action control according to TEC (theory of event coding). Psychological Research PRPF, 73(4), 512-526.

Irwin, D. E. (1991). Information integration across saccadic eye movements. Cognitive Psychology, 23(3), 420-456.

Irwin, D. E., \& Gordon, R. D. (1998). Eye movements, attention and trans-saccadic memory. Visual Cognition, 5(1/2), 127-155.

Irwin, D. E., Zacks, J. L., \& Brown, J. S. (1990). Visual memory and the perception of a stable visual environment. Perception \& Psychophysics, 47(1), 35-46.

Jarvik, M. E. (1956). Simple color discrimination in chimpanzees: Effect of varying contiguity between cue and incentive. Journal of Comparative and Physiological Psychology, 49(5), 492-495.

JASP Team (2017). JASP (Version 0.8.5) [Computer software].

Kahneman, D., Treisman, A., \& Gibbs, B. J. (1992). The reviewing of object files: Object-specific integration of information. Cognitive Psychology, 24(2), 175-219.

Kehoe, E. J., Cool, V., \& Gormezano, I. (1991). Trace conditioning of the rabbit's nictitating membrane response as a function of CS-US interstimulus interval and trials per session. Learning and Motivation, 22(3), 269-290.

Kiesel, A., \& Hoffmann, J. (2004). Variable action effects: Response control by context-specific effect anticipations. Psychological Research, 68, 155-162.

Kim, D., Seitz, A. R., \& Watanabe, T. (2015). Visual perceptual learning by operant conditioning training follows rules of contingency. Visual Cognition, 23(1/2), 147-160.

Kleiner, M., Brainard, D., \& Pelli, D. (2007). What's new in Psychtoolbox-3. Perception Abstract Supplement, 36(14), 1.

Kruschke, J. K. (2003). Attention in learning. Current Directions in Psychological Science, 12(5), 171-175. 
Madelain, L., Paeye, C., \& Wallman, J. (2011). Modification of saccadic gain by reinforcement. Journal of Neurophysiology, 106(1), 219 232

Marchand, A. R., \& Kamper, E. (2000). Time course of cardiac conditioned responses in restrained rats as a function of the trace CS-US interval. Journal of Experimental Psychology: Animal Behavior Processes, 26(4), 385-398.

Marx, S., \& Einhäuser, W. (2015). Reward modulates perception in binocular rivalry. Journal of Vision, 15(1), 1-11.

McConkie, G. W., \& Currie, C. B. (1996). Visual stability across saccades while viewing complex pictures. Journal of Experimental Psychology: Human Perception and Performance, 22(3), 563-581.

Montagnini, A., \& Chelazzi, L. (2005). The urgency to look: Prompt saccades to the benefit of perception. Vision Research, 45(27), 3391-3401.

Moore, C. M., Mordkoff, J. T., \& Enns, J. T. (2007). The path of least persistence: Object status mediates visual updating. Vision Research, 47(12), 1624-1630.

Noles, N. S., Scholl, B. J., \& Mitroff, S. R. (2005). The persistence of object file representations. Perception \& Psychophysics, 67(2), 324 334.

Oostwoud, W. L., Marshall, L., \& Bays, P. (2015). Visual updating across saccades by working memory integration. Journal of Vision, 15(12), 785-785.

O'Regan, J. K., \& Noë, A. (2001). A sensorimotor account of vision and visual consciousness. Behavioral and Brain Sciences, 24(05), 939 973.

Paeye, C., Collins, T., \& Cavanagh, P. (2017). Transsaccadic perceptual fusion. Journal of Vision, 17(1), 1-14.

Paeye, C., \& Madelain, L. (2011). Reinforcing saccadic amplitude variability. Journal of the Experimental Analysis of Behavior, 95(2), 149-162.

Paeye, C., \& Madelain, L. (2014). Reinforcing saccadic amplitude variability in a visual search task. Journal of Vision, 14(13), 1-20.

Paeye, C., Schütz, A., \& Gegenfurtner, K. (2016). Visual reinforcement shapes eye movements in visual search. Journal of Vision, 16(10), $1-15$.

Pavlov, I. (1927). Conditioned reflexes. London: Oxford University Press.

Pelli, D. (1997). The VideoToolbox software for visual psychophysics: Transforming numbers into movies. Spatial Vision, 10, 437-442.

Poth, C. H., Herwig, A., \& Schneider, W. X. (2015). Breaking object correspondence across saccadic eye movements deteriorates object recognition. Frontiers in Systems Neuroscience, 9(176), 1-10.

Prime, S. L., Niemeier, M., \& Crawford, J. D. (2006). Transsaccadic integration of visual features in a line intersection task. Experimental Brain Research, 169(4), 532-548.

Quinn, W. G., Harris, W. A., \& Benzer, S. (1974). Conditioned behavior in Drosophila melanogaster. Proceedings of the National Academy of Sciences, 71(3), 708-712.

Rescorla, R., \& Wagner, A. (1972). A theory of Pavlovian conditioning: Variations in the effectiveness of reinforcement and nonreinforcement. In A. H. Black \& W. F. Prokasy (Ed.), Classical conditioning II: Current research and theory (pp. 64-99). New York: Appleton-Century-Crofts.

Richard, A. M., Luck, S. J., \& Hollingworth, A. (2008). Establishing object correspondence across eye movements: Flexible use of spatiotemporal and surface feature information. Cognition, 109(1), 6688.
Rolfs, M., Jonikaitis, D., Deubel, H., \& Cavanagh, P. (2011). Predictive remapping of attention across eye movements. Nature Neuroscience, 14(2), 252-256.

Ross, J., Morrone, M. C., Goldberg, M. E., \& Burr, D. C. (2001). Changes in visual perception at the time of saccades. Trends in Neurosciences, 24(2), 113-121.

Schneider, W. X. (2013). Selective visual processing across competition episodes: A theory of task-driven visual attention and working memory. Philosophical Transactions of the Royal Society B, $368(1628), 1-13$.

Schultz, W. (2006). Behavioral theories and the neurophysiology of reward. Annual Review of Psychology, 57, 87-115.

Schütz, A. C., Kerzel, D., \& Souto, D. (2014). Saccadic adaptation induced by a perceptual task. Journal of Vision, 14(5), 1-19.

Seitz, A. R., Kim, D., \& Watanabe, T. (2009). Rewards evoke learning of unconsciously processed visual stimuli in adult humans. Neuron, 61(5), 700-707.

Seitz, A. R., Nanez, J. E., Holloway, S. R., Koyama, S., \& Watanabe, T. (2005). Seeing what is not there shows the costs of perceptual learning. Proceedings of the National Academy of Sciences of the United States of America, 102(25), 9080-9085.

Seitz, A. R., \& Watanabe, T. (2003). Psychophysics: Is subliminal learning really passive? Nature, 422(6927), 36.

Seitz, A. R., \& Watanabe, T. (2005). A unified model for perceptual learning. Trends in Cognitive Sciences, 9(7), 329-334.

Shafir, S. (1996). Color discrimination conditioning of a wasp, Polybia occidentalis (Hymenoptera: Vespidae). Biotropica, 28(2), 243-251.

Shin, Y. K., Proctor, R. W., \& Capaldi, E. J. (2010). A review of contemporary ideomotor theory. Psychological Bulletin, 136(6), 943-974.

Sidman, M. (2008). Reflections on stimulus control. The Behavior Analyst 31 (2), 127-135.

Tas, A. C., Moore, C. M., \& Hollingworth, A. (2012). An objectmediated updating account of insensitivity to transsaccadic change. Journal of Vision, 12(11), 1-18.

Valsecchi, M., \& Gegenfurtner, K. R. (2016). Dynamic re-calibration of perceived size in fovea and periphery through predictable size changes. Current Biology, 26(1), 59-63.

Weiß, K., \& Herwig, A. (2015). Where triangles become circular: The impact of transsaccadic predictions on shape perception depends on retinal eccentricity. Journal of Eye Movement Research, 8(4), 191.

Weiß, K., Schneider, W. X., \& Herwig, A. (2014). Associating peripheral and foveal visual input across saccades: A default mode of the human visual system? Journal of Vision, 14(11), 1-15.

Wolf, C., \& Schütz, A. C. (2015). Trans-saccadic integration of peripheral and foveal feature information is close to optimal. Journal of Vision, 15(16), 1-18.

Wolfe, B. A., \& Whitney, D. (2015). Saccadic remapping of objectselective information. Attention, Perception, \& Psychophysics, 77(7), 2260-2269.

Wurtz, R. H. (2008). Neuronal mechanisms of visual stability. Vision Research, 48(20), 2070-2089.

Xu-Wilson, M., Zee, D. S., \& Shadmehr, R. (2009). The intrinsic value of visual information affects saccade velocities. Experimental Brain Research, 196(4), 475-481.

Zimmermann, E., Born, S., Fink, G. R., \& Cavanagh, P. (2014). Masking produces compression of space and time in the absence of eye movements. Journal of Neurophysiology, 112(12), 3066-3076.

Zimmermann, E., Fink, G., \& Cavanagh, P. (2013). Perifoveal spatial compression. Journal of Vision, 13(5), 1-21. 